\title{
Reutilização da água gerada pelos ares-condicionados: análise dos benefícios através da modelagem computacional
}

O artigo apresenta a modelagem e desenvolvimento de um modelo de simulação para avaliar o reaproveita da água gerada pelos ares-condicionados de uma Instituição de Ensino Superior (IES) pública. Para a construção do modelo (definição de variáveis e suas interrelações) utilizou-se por base teórica as pesquisas bibliográficas e observações do processo da geração da água. Dentre os aspectos analisados, está a redução do impacto financeiro, em função da redução do gasto com o consumo de água potável. Para avaliar as possibilidades, foram gerados três cenários: um cenário baseado na situação atual; outro denominado Cenário Moderado; e, por fim, o cenário otimista. Os resultados obtidos através da simulação demonstram que o processo de reaproveitamento traz um significativo ganho financeiro. O horizonte de tempo simulado foi de dez anos e foi utilizado o software Vensim para o desenvolvimento da simulação.

Palavras-chave: Ar-condicionado; Modelagem Computacional; Vensim.

\section{Reuse of water generated by air conditioners: benefit analysis through computer modeling}

The article presents the modeling and development of a simulation model to evaluate the reuse of water generated by the air conditioners of a public Higher Education Institution (HEI). For the construction of the model (definition of variables and their interrelationships) the theoretical basis was the bibliographic research and observations of the process of water generation. Among the aspects analyzed is the reduction of the financial impact, due to the reduction in the consumption of drinking water. To evaluate the possibilities, three scenarios were generated: a scenario based on the current situation; another called Moderate Scenario; and finally the optimistic scenario. The results obtained through the simulation show that the reuse process brings a significant financial gain. The simulated time horizon was ten years and the Vensim software was used to develop the simulation.

Keywords: Air-conditioning; Computational modeling; Vensim.

Topic: Pesquisa Operacional

Reviewed anonymously in the process of blind peer.
Received: 09/07/2019

Approved: 10/09/2019
Glauco Oliveira Rodrigues

Universidade Federal de Santa Maria, Brasi http://lattes.cnpq.br/9943217673304581

glaucop10@redes.ufsm.br

Jardel Schneider

Universidade Federal de Santa Maria, Brasi http://lattes.cnpq.br/9393503631677257 glaucorodriguesp10@gmail.com

Fernando Pires Barbosa

Universidade Federal de Santa Maria, Brasil http://lattes.cnpq.br/1352060153158116 glaucorodriguesp10@gmail.com
Paulo Roberto Langwinski

Universidade Federal de Santa Maria, Brasil http://lattes.cnpq.br/6357862861317072 glaucorodriguesp10@gmail.com

Douglas Soares

Universidade de São Paulo, Brasil

http://lattes.cnpq.br/3968855358932741

glaucorodriguesp10@gmail.com
Referencing this:

RODRIGUES, G. O.; SCHNEIDER, J.; BARBOSA, F. P.; LANGWINSKI, P. R.; SOARES, D.. Desenvolvimento de competências: a percepção de gestores sobre técnicos administrativos em uma universidade federal. Revista Brasileira de Administração Científica, v.10, n.3, p.71-79, 2019. DOI: http://doi.org/10.6008/CBPC2179-684X.2019.003.0006 


\section{INTRODUÇÃO}

A escassez de água potável no mundo é um problema que exige atenção dos pesquisadores do planeta. Uma definição para o uso racional da água pode ser definida por práticas, técnicas e tecnologias que propiciam a melhoria da eficiência do seu uso, sendo que devido à sua escassez contínua. Alguns processos, como o de reaproveitamento da água, têm crescido nos últimos anos, além de que a escassez da água faz com que ela se torne mais valiosa e sua economia também gera economia financeira para seus usuários. Empresas e pessoas físicas estão cada vez mais preocupadas com questões ambientais, procurando formas de reciclar a água utilizada em seus prédios ou ainda coletando água da chuva para aproveitamento em limpeza, jardinagem e esgoto.

A sustentabilidade hídrica é um tema abordado continuamente nos objetos de estudo. Para isso deve-se aplicar em larga escala o desenvolvimento sustentável. O desenvolvimento sustentável é definido como sendo o desenvolvimento capaz de suprir as necessidades da geração atual, garantindo a capacidade de atender as necessidades das futuras gerações.

A problemática da água está inserida em um amplo contexto em que vários fatores afetam a perda da eficiência no seu ciclo hidrológico, contribuindo para a sua escassez. As causas são problemas diversos, como a crescente urbanização sem planejamento da infraestrutura urbana, no qual a ausência de abastecimento de água e saneamento acarreta também, por consequência, agravos à saúde pública. Atrelado a está questão está o reuso da água. É importante observar que esta prática está relacionada a dois aspectos: Como instrumento para redução do consumo de água, como um controle de demanda e como recurso hídrico complementar.

Uma possibilidade de aplicação da sustentabilidade seria coletar a água gerada por arescondicionados de Instituições de Ensino Superior e reutilizar a água para o seu consumo interno, como limpeza, jardinagem e banheiros. Para isso, uma infraestrutura de coleta deverá ser instalada. A partir desta ideia, o presente artigo tem como objetivo gerar modelos computacionais para analisar o benefício ambiental e financeiro do reuso da água gerada por ares-condicionados de uma IES do sul do Brasil.

Quanto à estrutura, após esse capítulo introdutório, apresenta-se o referencial teórico. Em seguida, consta o método de pesquisa adotado. Na sequência, segue a descrição do desenvolvimento do modelo e resultados e, posteriormente, encerra-se com as considerações finais acompanhada de recomendações para pesquisas futuras.

\section{METODOLOGIA}

Para o desenvolvimento deste artigo, foi utilizada como metodologia a modelagem computacional, a manipulação do modelo desenvolvido foi feita atrás da simulação computacional. A modelagem consiste na elaboração de representações de um sistema real, chamadas de modelos (ANDRADE et al., 2006). A simulação é a operação sobre um modelo de interesse (PRADO, 2010), enquanto que a simulação computacional, especificamente, é aquela simulação que utiliza um computador para ser realizada (CHWIF 
et al., 2015), trata-se de uma técnica que permite transcrever um sistema real para um ambiente computacional utilizando recursos oferecidos por computadores (PRADO, 2010).

O método é constituído pelos seguintes passos: estudos exploratórios em artigos científicos, relatórios e orientações técnicos, bem como da legislação referente ao assunto; coleta de informações e documentos, entrevistas e reuniões com os servidores da Instituição parceira ao estudo; definição dos cenários à serem analisados, desenvolvimento das possíveis soluções, definição das variáveis, seus relacionamentos e equitações; implementação computacional da solução idealizada no simulador Vensim; e avaliação dos resultados dos cenários.

Os dados primários para as entradas do modelo foram coletados na instituição analisada, para desenvolvimento e avaliação do referido modelo. Para a definição das variáveis componentes do modelo, foram realizadas entrevistas com stakeholders da instituição (para garantir maior fidedignidade ao estudo), bem como levantamento bibliográfico em artigos e relatórios técnicos.

\section{DISCUSSÃO TEÓRICA}

\section{Sustentabilidade e recursos hídricos}

A sustentabilidade recebe atenção de estudiosos do mundo todo, por meio do desenvolvimento de pesquisas e novas abordagens teóricas referentes a estratégias de produção mais limpa, controle da poluição, ecoeficiência, gestão ambiental, responsabilidade social, ecologia industrial, investimentos éticos, economia verde, eco design, reuso, consumo sustentável e resíduos zero.

Porém, os conceitos de sustentabilidade e Desenvolvimento Sustentável - DS ainda são mal compreendidos, e em muitos casos, são tratados como sinônimos. Mas nem todos os que pesquisam esses conceitos os veem assim. Sustentabilidade é a capacidade de um sistema humano, natural ou misto resistir ou se adaptar à mudança endógena ou exógena por tempo indeterminado, e, além disso, o DS é uma via de mudança intencional e melhoria que mantém ou aumenta esse atributo do sistema, ao responder as necessidades da população presente. Numa primeira visão, o DS, é o caminho para se alcançar a sustentabilidade, isto é, a sustentabilidade é o objetivo final, de longo prazo.

A sustentabilidade, para Elkington (1994), é o equilíbrio entre três pilares: ambiental, econômico e social. A perspectiva de que as empresas devem contribuir de forma progressiva com a sustentabilidade surge do reconhecimento de que os negócios precisam de mercados estáveis, e que devem possuir habilidades tecnológicas, financeiras e de gerenciamento necessário para possibilitar a transição rumo ao desenvolvimento sustentável (ELKINGTON, 2001). Tem-se, portanto, uma segunda visão, diferente da primeira: o DS é objetivo a ser alcançado e a sustentabilidade é o processo para atingir o DS.

Apesar de existirem as duas visões, esta pesquisa volta-se para a sustentabilidade. Ressalta-se a existência de uma variedade de pesquisas sobre o assunto, buscando compreender o conceito de sustentabilidade seja ela como um processo ou um objetivo final. Nesse sentido, torna-se importante ter uma noção clara do que se entende por sustentabilidade ou a falta dela. 
O termo sustentabilidade é utilizado, mas pouco explicado. É de natureza conceitual, mal compreendido. Trata-se de um acessório de moda ou um senso comum. Há inconsistente interpretação e aplicação, alto grau de ambiguidade do conceito, incluindo uma percepção de incompletos problemas de pobreza, degradação ambiental e o papel do crescimento econômico. E a situação não tem melhorado até então, continua sendo um slogan popular e brilhante.

Para aplicar a sustentabilidade hídrica, deve-se atentar para gestão hídrica. A gestão de recursos hídricos no Brasil é orientada pela Política Nacional de Recursos Hídricos (PNRH), instituída pela Lei no 9.433, de 8 de janeiro de 1997, conhecida como Lei das Águas, a qual criou o Sistema Nacional de Gerenciamento de Recursos Hídricos (SINGREH). Desta forma, aconselha-se uma gestão integrada e participativa, que visa principalmente garantir a disponibilidade de água à atual e às próximas gerações, em padrões de qualidade adequados aos respectivos usos.

Para a implementação da PNRH, foi criada Agência Nacional de Águas (ANA) instituída pela Lei no 9.984 de 2000. Esta se caracteriza como um órgão regulador, com vínculo ao Ministério do Meio Ambiente (MMA), responsável, sobretudo pela implementação, operacionalização, controle e avaliação dos instrumentos de gestão e outras funções inerentes aos recursos hídricos (ANA, 2011).

Os Comitês de Bacias Hidrográficas têm como atribuição legal determinar sobre a gestão das águas em conformidade com o poder público, e desempenham papel importante neste sistema participativo de gestão. Dentre outras competências no âmbito da sua área de atuação, são responsáveis pela aprovação da adequada aplicação dos Planos de Recursos Hídricos da bacia, que permitem integrar e articular os demais instrumentos da Política (ANA, 2011).

\section{Modelagem computacional}

Modelagem Computacional é uma área de conhecimento multidisciplinar que trata da aplicação de modelos matemáticos e técnicas da computação à análise, compreensão e estudo da fenomenologia de problemas complexos em áreas tão abrangentes quanto às engenharias, ciências exatas, biológicas, humanas, economia e ciências. São representações incompletas e mais simples do que o objeto ou sistema em questão. Para Andrade et al. (2006), a modelagem computacional é uma das ferramentas do pensamento sistêmico que adicionam aprendizado ao processo e por meio da mesma constroem-se micromundos do sistema real.

Para pesquisadores como Andrade (2006), Maani et al. (2000), entre outros, os modelos podem apresentar dois tipos de modelagem, distintamente chamados de soft e hard. A modelagem soft refere-se a abordagens conceituais que buscam maior realismo, pluralismo e uma intervenção mais holística que a modelagem hard. Os conceitos soft e hard são também relacionados às ideias de qualitativo e quantitativo, respectivamente. Um modelo computacional é construído com basicamente quatro componentes: Estoques, fluxos, conectores e auxiliar. 


\section{Componentes do modelo}

Os estoques são variáveis de estado, e podem ser considerados como repositórios para acumular ou armazenar para outros elementos do sistema (DEATON et al., 2000). Tem como principal importância fornecer uma visão de como está o sistema em qualquer instante de tempo, mudanças nos estoques não são instantâneas, elas demandam certo tempo e ocorrem devido à ação dos fluxos. Por sua vez, os fluxos são variáveis de ação, pois elas podem aumentar ou diminuir o volume determinado no estoque. Produz crescimento ou redução dos estoques, o movimento de materiais e a informação dentro do sistema.

As variáveis auxiliares são componentes para a realização de operações algébricas, que processam informações a respeito dos estoques e fluxos ou representam fontes de informação externas ao sistema (BLOIS et al., 2008). Existem os auxiliares que também podem modificar outras variáveis auxiliares, são muitos utilizados para modelar as informações e não o fluxo físico, podendo alterar instantaneamente sem atrasos.

Por fim, existem os conectores, que são os que representam as interrelações entre todos os componentes, ou seja, são essas interligações que ligam os componentes que formam a expressão matemática (STRAUS, 2010). Possuem função de links de informação que descrevem a relação entre estoques, fluxos e auxiliares. A figura 1 demonstra cada um dos componentes de um modelo de Dinâmica de Sistemas.

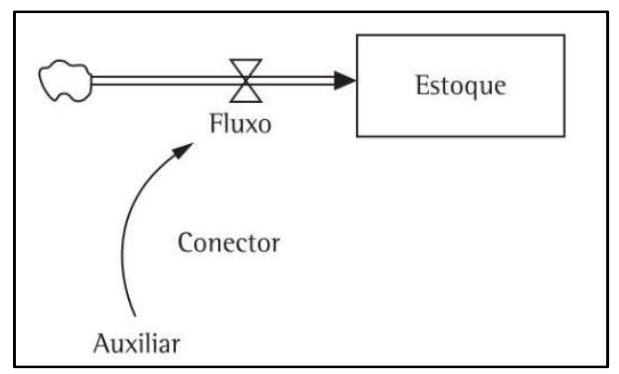

Figura 1: Componentes do modelo.

\section{Desenvolvimento do modelo e resultados}

Diante da crise econômica vivenciada pelo Brasil, cortes orçamentários acontecem com mais frequência, cabendo às Instituições acharem estratégias para diminuir os custos necessários para manter o seu pleno funcionamento. Dentre os gastos necessários para suprir e atender as necessidades dos usuários de uma Instituição de Ensino Superior, está a água. Devido a tal, torna-se viável a proposta do desenvolvimento sustentável, que é o desenvolvimento capaz de suprir as necessidades usuários da situação atual, garantindo a capacidade de atender as necessidades das gerações futuras.

Os aparelhos de ar-condicionado, que são utilizados em larga escala nos prédios da IES, geram gotejamento de água, derivada da umidade do ar condensado quando o aparelho resfria o ar do ambiente interno. $\mathrm{O}$ artigo visa analisar o aproveitamento da água gerada pelos ares condicionados. De acordo com Mota (2011), em média, um ar-condicionado com 12000BTUs gera em torno de 300 mililitros de água por hora ou 0,3 litros por hora, enquanto os de 9000BTUs geram a metade desta quantia. Para reforçar este dado, foi realizada uma coleta em aproximadamente dez ares-condicionados da IES parceira do estudo, o 
quadro 1 apresenta a quantidade de ares-condicionados. Os aparelhos ficam ligados em média 14 horas diários e aproximadamente 22 dias por mês.

Quadro 1: Dados Modelagem.

\begin{tabular}{|c|c|}
\hline Potência Ar Condicionado & Quantidade \\
\hline 48000 & 10 \\
\hline 36000 & 40 \\
\hline 24000 & 384 \\
\hline 22000 & 1032 \\
\hline 18000 & 1324 \\
\hline 12000 & 1111 \\
\hline 9000 & 424 \\
\hline 7500 & 101 \\
\hline 7000 & 55 \\
\hline
\end{tabular}

O custo do $\mathrm{m}^{3}$ da água varia bastante, com a Companhia Riograndense de Saneamento (CORSAN) cobrando cerca de R\$0,005 pelo litro da água. A média no Brasil do litro da água fica em torno dos R\$0,0035. Para reforçar o custo do consumo da água da IES base de estudo, foram coletadas 48 contas de água dos diferentes prédios da IES, possibilitando aos pesquisadores entenderem a lógica do consumo/gasto da água da instituição.

A figura 2 representa o modelo desenvolvido para armazenar a lógica de captação da água gerada pelos ares-condicionados. Neste modelo existem apenas variáveis auxiliares, a lógica das variáveis responsáveis por armazenar a quantidade de água por ar condicionado é igual para todos, por exemplo: A variável 'Água4800BTU' recebe a interação das variáveis auxiliares 'Geração 4800BTU' e 'Qt ar 4800BTU'. A variável central neste modelo é a 'Total Água Gerada', nesta variável a quantidade de litros de todos os arescondicionados é somado.

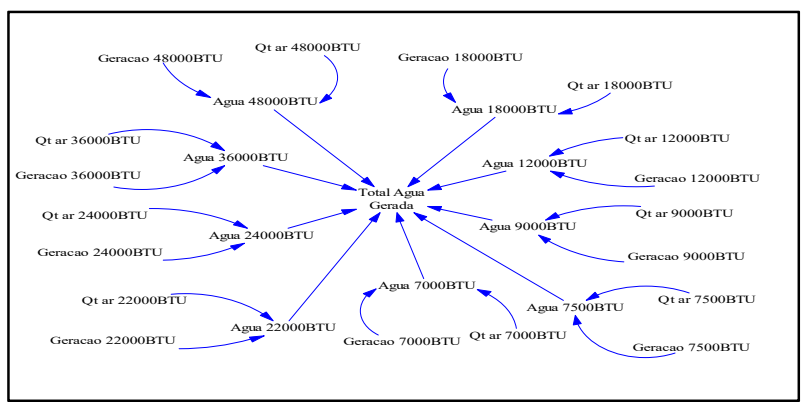

Figura 2: Modelo Computacional I.

A figura 3 representa o modelo desenvolvido para armazenar o resultado deste artigo. Neste modelo o custo da Instituição com a água é calculado. As três variáveis auxiliares 'ConsumoLimpeza', 'ConsumoBanheiro' e 'ConsumoJardinagem' são a entrada da variável do tipo 'Rate', chamada 'ConsumoLimpeza'. As variáveis 'Rate' indicam os valores que vão ser somados ou subtraídos nas variáveis acumuladores ou de estoque. A interação das variáveis auxiliares 'Custo $\mathrm{m}^{2 \prime}$ e 'Total $\mathrm{m}^{2 \prime}$ resultam no valor de entrada da variável 'Rate' 'Custolnfraestrutura'.

Por fim a variável auxiliar citada no modelo anterior 'Total Água Gerada' interagindo com a variável 'Taxa de Aproveitamento' serão os dados da Rate variável 'AguaAproveitada'. Vale ressaltar que a variável 'Taxa de Aproveitamento' representa a proposta dos autores, através dela será possível a geração de 
diferentes cenários de simulações. Por fim a variável de estoque 'Custo Água' recebe a interação das 'Rate' variáveis junto com a última variável RATE chamada 'Preço Litro', que mensurará o preço do litro de água potável paga pela IES. As equações principais dos modelos estão expostas no quadro 2.

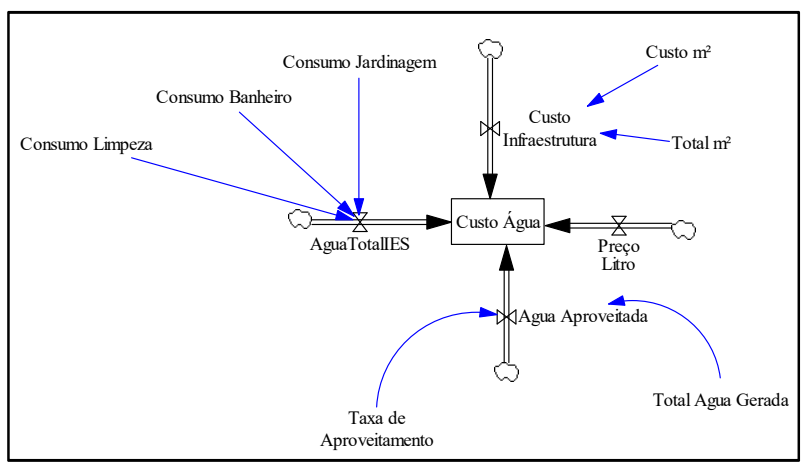

Figura 3: Modelo Computacional II.

Quadro 2: Equações.

Total Água = Água 12000BTUs+Água 18000BTUs+Água 22000BTUs+Água 24000BTUs+Água 36000BTUs+Água 48000BTUs+Água 7000BTUs+Água 7500BTUs+Água9000BTUs

AguaTotalIES = Consumo Banheiro+Consumo Jardinagem+Consumo Limpeza

Custo Infraestrutura $=$ Custo $\mathrm{m}^{2 *}$ Total $\mathrm{m}^{2}$

Água Aproveitada = Taxa de Aproveitamento*Total Água Gerada

Custo Água = ((AguaTotalIES-Agua Aproveitada $) *$ Preço Litro $)+$ Custo Infraestrutura

Para possibilitar a análise e a comparação das propostas, foram gerados 3 cenários. O cenário atual, onde não existe o reaproveitamento da água gerada pelos ares-condicionados; o cenário mediano, onde 25\% da água será aproveitada; e o cenário otimista, onde $60 \%$ da água será aproveitada. Lembrando que não existe como aproveitar $100 \%$ da água, já que boa parte dela evapora.

Definidos os cenários para a realização do experimento com o uso do modelo, foram executadas as simulações. Para a execução das simulações, foi utilizado o simulador Vensim em uma estrutura computacional com processador Intel Core (i5 2450) de 2,5Ghz, 4Gb de memória RAM e o tempo de execução da simulação dos três cenários foi na ordem de milionésimos de segundo. $O$ tempo de simulação pegou o ano de 2018 como ano inicial, onde os dados foram coletados, e 2027, como ano final da pesquisa. O modelo possibilitará aos interessados gerarem outras simulações, podendo criar diferentes cenários já que o modelo foi construído com o objetivo de ofertar melhores decisões de reaproveitamento da água gerada pelos ares condicionados.

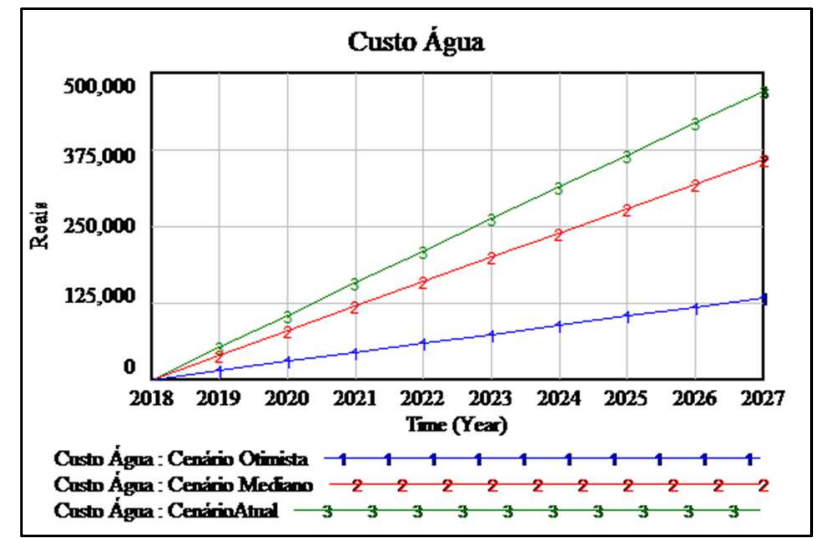

Figura 4: Resultado da Simulação. 
O primeiro dado-análise é que se fosse aproveitado toda a água gerada pelos ares-condicionados da Instituição de Ensino Superior, seriam coletados, aproximadamente, 25.027.200L de água em dez anos de uso, reafirmando a importância da pesquisa e de sua aplicação, já que esta quantidade é de apenas uma IES. Para verificar o gasto gerado pelo consumo de água, gerou-se o gráfico apresentado na figura 4.

Nota-se o cenário atual como o representante do pior cenário financeiro, gastando aproximadamente $\mathrm{R} \$ 470 \mathrm{mil}$ em dez anos de simulação. O cenário mediano apresenta melhor desempenho, gastando aproximadamente $\mathrm{R} \$ 112$ mil a menos que o cenário atual. $\mathrm{O}$ cenário que apresenta a melhor economia para a IES é o cenário otimista, que gastará aproximadamente $\mathrm{R} \$ 132 \mathrm{mil}$, totalizando uma diferença de $\mathrm{R} \$ 337$ mil comparados aos cenários atuais. O cenário otimista ainda apresenta $\mathrm{R} \$ 219$ mil de economia quando comparado a outra proposta dos modeladores. Observa-se que se aproveitado maior quantidade de água a IES poderá obter maior economia financeira.

\section{CONSIDERAÇÕES FINAIS}

A preocupação com a escassez de água se estende a muitas das regiões metropolitanas brasileiras. Embora o Brasil disponha de grande porcentagem dos recursos hídricos mundiais, muitas regiões convivem com recursos hídricos da ordem de duzentos metros cúbicos por habitante por ano, gerando condições críticas de abastecimento e conflitos no uso da água. É necessário investir em programas objetivos de medição, avaliação e pesquisa, bem como em programas de motivação e treinamento pessoal. Portanto, o objetivo principal deste artigo foi o desenvolvimento, verificação, avaliação e experimento de modelos de simulação computacional, com o propósito de avaliar grupos de cenários para o reaproveitamento da água gerada por ares-condicionados.

Para o desenvolvimento do modelo de simulação, levou-se em consideração o conceito que modelos são compostos por variáveis de estoque, fluxo, ambas variáveis endógenas. Um dos objetivos centrais da metodologia de modelagem computacional é ter um modelo que consiga simular o comportamento real. Ou seja, a fonte dos problemas em um sistema seja uma parte inerente do modelo desenvolvido.

A metodologia auxiliou a mapear as estruturas do sistema desenvolvido, procurando examinar sua inter-relação em contexto amplo. Através da simulação desenvolvida, a dinâmica aplicada pretende compreender como o sistema em foco evolui no tempo e como as mudanças em suas partes afetam o seu comportamento. A partir dessa compreensão, foi possível diagnosticar e prognosticar o sistema, além de possibilitar simular mais cenários no tempo.

Com relação específica aos resultados obtidos, para os cenários avaliados, o cenário otimista apresentou os melhores resultados ofertando uma economia de aproximadamente $\mathrm{R} \$ 33.000,00$ ao ano. $O$ cenário mediano também ofertará economia financeira quando comparado ao cenário atual, reforçando a importância de aproveitar a água gerada pelos ares-condicionados da Instituição de Ensino Superior parceira da pesquisa.

A relevância disso não se limita apenas à criação de uma alternativa para a utilização mais consciente de água, mas também ao fato de se ter uma alternativa para o uso da água gerada pelos ares-condicionados. 
A água, conforme descrito anteriormente, é um recurso que necessita ser cada vez mais racionalmente utilizado, visto que sua quantidade útil disponível diminui a cada ano.

Uma das principais limitações desta investigação refere-se ao fato de o modelo ter sido desenvolvido para analisar uma instituição pública, o que poderá impedir a generalização dos achados para outras IES privadas. Outra limitação se refere no custo da água, já que existe discrepância nos valores anuais pagos em água pela IES. Como trabalhos futuros pretendem-se acrescentar variáveis ao modelo, como por exemplo, o custo para criar os dispositivos de coleta de água.

\section{REFERÊNCIAS}

ANA. Agência Nacional de Águas. O Comitê de Bacia Hidrográfica: o que é e o que faz. Brasília: ANA, 2011.

ANDRADE, A. L.; SELEME, A.; RODRIGUES, L. H.; SOUTO, R.. Pensamento Sistêmico: caderno de campo: o desafio da mudança sustentada nas organizações e na sociedade. Porto Alegre: Bookman, 2006.

BLOIS, D. H.; SOUZA, J. C.. Cenários Prospectivos e a Dinâmica de Sistemas: proposta de um modelo para o setor calçadista. Revista de Administração de Empresas, v.48, n.3, 2008.

CHWIF, L.; MEDINA, A. C.. Modelagem e Simulação de eventos discretos: Teoria e Aplicação. 4 ed. Rio de Janeiro: Elsevier, 2015.

DEATON, M. L.; WINEBRAKE, J. J.. Dynamic Modelling of Environmental Systems. Berlin: Springer-Verlag, 2000.

ELKINGTON, J.. Enter the triple botom line. In: HENRIQUES, A.; RICHARDSON, J.. The triple botom line, does it all add up?: Assessing the sustainability of business and CSR. London: Earthscan Publications, 2004. p.1-16.

GIL, A. C.. Como elaborar projetos de pesquisa. 5 ed. São Paulo: Atlas, 2010.

LAW, A. M.. Simulation Modeling and Analysis. 5 ed. Nova York: McGraw-Hill, 2015.

MARCIAL, E. C.; GRUMBACH, R. J. S.. Cenários prospectivos: como construir um futuro melhor. 3 ed. Rio de Janeiro: FGV 2005.

MOTA, T. R.. Utilização da água de sistemas de ar condicionado visando o desenvolvimento sustentável. Maringá: Universidade Estadual de Maringá, 2011.

STRAUS, L. M.. Um modelo em dinâmica de sistemas para o ensino superior. Dissertação (Mestrado em Administração) Universidade Federal do Rio Grande do Sul, Porto Alegre 2010.

A CBPC - Companhia Brasileira de Produção Científica (CNPJ: 11.221.422/0001-03) detém os direitos materiais desta publicação. Os direitos referem-se à publicação do trabalho em qualquer parte do mundo, incluindo os direitos às renovações, expansões e disseminações da contribuição, bem como outros direitos subsidiários. Todos os trabalhos publicados eletronicamente poderão posteriormente ser publicados em coletâneas impressas sob coordenação da Sustenere Publishing, da Companhia Brasileira de Produção Científica e seus parceiros autorizados. Os (as) autores (as) preservam os direitos autorais, mas não têm permissão para a publicação da contribuição em outro meio, impresso ou digital, em português ou em tradução. 